protonated (Oseroff and Callendar, Biochemistry, 13, 4243; 1974).

The difficult question to resolve is which species are primary photoproducts leading to a biochemical event and which competing by-products. Since the quantum efficiencies of formation of these species are not known and since the quantum efficiencies of the photobiological processes of rhodopsin and bacteriorhodopsin are less than unity, this question remains open. In view of the function of bacteriorhodopsin it is worth examining the hypothesis that the loss of a proton from the excited Schiff base may be the primary photoprocess. Involving the motion of a proton along the $\mathrm{N}-\mathrm{H}$ vibrational coordinate this transfer will be exceedingly fast, within the time of a single vibration. All that is required is a change in the basicity of the $\mathrm{N}$ atom in the excited state. If this were followed by a slower thermal isomerism of the polyene chain the role of the protein in selecting the course of the isomerism becomes more comprehensible. One possible test of this hypothesis might be made by measuring the quantum efficiencies of the photoprocesses of rhodopsin and bacteriorhodopsin after deuteration of the Schiff base link. A pronounced isotope effect should be seen only if proton transfer is involved in the primary photoprocess.

\section{Globin synthesis: rates and ratios}

\section{from Pamela Hamlyn}

IT has been known for several years that the $\alpha$ and $\beta$ globin chains of mammalian haemoglobin are synthesised on different polysomes and yet their production is controlled in such a way that nearly equal amounts of each are produced. The means by which these two independent events are synchronised is the subject of some interest for it seems possible that this mechanism may be one of general importance in the control of synthesis of proteins composed of two or more subunits.

Lodish and his co-workers have made several contributions to the study of globin synthesis control and recently Lodish (Nature, 251, 385; 1975) has derived a simple kinetic rate equation for initiation and elongation of polypeptide chains which, although based on $\alpha$ and $\beta$ globin synthesis, may be applicable to eukaryotes in general. A result of these calculations is the prediction that any reduction in the rate of polypeptide chain initiation will lead to preferential inhibition of translation of mRNAs with lower rate constants for polypeptide initiation. Lodish has argued that $\alpha$ mRNA has a lower initiation constant than $\beta$ mRNA. Based on the observation that $\alpha$ mRNA is found in polysomes averaging three ribosomes, whereas $\beta$ mRNA polysomes have five ribosomes on average, he contends that since the rate of elongation is the same for $\alpha$ and $\beta$ chains then $\alpha$ mRNA must initiate protein synthesis only $60 \%$ as often as $\beta$ mRNA. (He suggests that balanced synthesis of the two globins is achieved by a 1.7 -fold excess of $\alpha$ mRNA over $\beta$ mRNA.) Globin synthesis, therefore, provides an experimental situation to test the predictions of his model. Accordingly Lodish has added inhibitors of protein synthesis initiation to a reticulocyte lysate and measured the effect on the ratio of $\alpha$ and $\beta$ globin synthesised. In all cases he found that it was $\alpha$ mRNA translation that was preferentially inhibited as predicted by his model.

McKeehan, working at the Basel Institute for Immunology, has reported the results of a systematic study of globin synthesis in the reticulocyte lysate in which each of the components of the synthesis was varied in turnkeeping the others constant-and the effect on the $\alpha / \beta$ globin ratio measured (J. biol. Chem., 249, 6517; 1974). He found that increasing the concentration of globin mRNA resulted in a decrease in the $\alpha / \beta$ globin ratio, whereas increasing the concentration of 'initiation factors' (obtained from a salt wash of the small ribosomal subunit), or the concentration of the ribosomal subunits, resulted in an increase in the $\alpha / \beta$ globin ratio. When experiments were performed with separated subunits it was found that increasing the $40 \mathrm{~S}$ subunit relative to the $60 \mathrm{~S}$ gave an increase in the $\alpha / \beta$ globin ratio, whereas when the $60 \mathrm{~S}$ subunit was in excess then there was a small decrease in the $\alpha / \beta$ globin ratio.

McKeehan maintains that these results can best be explained using Lodish's assumptions, namely that $\beta$ globin mRNA initiates the synthesis of protein more efficiently than $\alpha$ mRNA, and also that there is more active $\alpha$ mRNA than $\beta$ mRNA bound to polysomes.

Consider for example when the concentration of $40 \mathrm{~S}$ ribosomal subunit is increased, all other components being held constant. The excess of small subunit means that $\alpha$ and $\beta$ mRNA are no longer in competition for initiation so that the superior efficiency of $\beta$ mRNA initiation is not involved in determining the $\alpha / \beta$ globin ratio produced in the reticulocyte lysate. Only the relative concentrations of the two messengers is effective in determining the globin ratio and since there is more $\alpha$ mRNA then the $\alpha / \beta$ globin ratio increases, as is observed experimentally.

Both Lodish and McKeehan make the point that these mechanisms for altering the ratio of $\alpha / \beta$ globin synthesised rely only on varying the relative amounts of the components of protein synthesis and do not require existence of $\alpha$ and $\beta$ mRNA specific initiation factors.

The results and predictions of Lodish and McKeehan go some way to defining the components in the mechanism of control of globin synthesis but do not explain how the feedback could operate in vivo. In other words, adding more 9S RNA to the constituents of protein synthesis may increase the relative amount of $\beta$ globin but how is the lack of $\beta$ globin communicated so that more 9S mRNA is made available to the ribosomes?

\section{rosy structural gene for xanthine dehydrogenase}

\section{from Benjamin Lewin}

What is a gene? This question has been resurrected by the present debate on the nature of the unit of gene expression in eukaryotes. At the heart of the discussion is the apparent discrepancy between the coding potential of the eukaryotic genome and its probable number of genes. This can be placed on a satisfactory quantitative basis really only with Drosophila, where a visible division of the genetic material into about 5,000 bands is evident in the polytene chromosomes of the salivary glands. That each band represents a single genetic function is suggested by the identification of individual lethal complementation groups with single bands in a region of the $\mathrm{X}$ chromosome (Judd, Shen and Kaufman, Genetics, $71,139-156 ; 1972$ ) and by the rough equality between the total number of sex-linked lethals and $\mathrm{X}$ chromosome bands (Hochman, Cold Spring Harb. Symp. quant. Biol., 38, 581-598; 1973) and between the total number of third chromosome late lethals and bands (Shearn, Genetics, 77, 115-126; 1974). But the average content of a band is about 30,000 base pairs, sufficient to code for 10-20 proteins of average size. Two extreme views can be taken of this situation: all genes may be identifiable by lethal mutations, so that each band contains only one protein-coding sequence, on average occupying about $10 \%$ of the DNA; or only a small minority of genes can be identified by lethal mutation, so that there may be many 'null loci' that are unidentifiable by mutation, in which case there might 